\title{
Geste technique, fait social total. Le technique est-il dans le social ou face à lui ?
}

Robert Cresswell

\section{(2) OpenEdition \\ Journals}

Édition électronique

URL : https://journals.openedition.org/tc/1576

DOI : $10.4000 /$ tc. 1576

ISSN : 1952-420X

Éditeur

Éditions de l'EHESS

Édition imprimée

Date de publication : 1 avril 2003

ISSN : 0248-6016

Référence électronique

Robert Cresswell, «Geste technique, fait social total. Le technique est-il dans le social ou face à lui ? ", Techniques \& Culture [En ligne], 40 | 2003, mis en ligne le 11 juillet 2006, consulté le 29 septembre

2022. URL : http://journals.openedition.org/tc/1576 ; DOI : https://doi.org/10.4000/tc.1576

Ce document a été généré automatiquement le 29 septembre 2022.

Tous droits réservés 


\title{
Geste technique, fait social total. Le technique est-il dans le social ou face à lui ?
}

\author{
Robert Cresswell
}

1 Le titre général de cet ensemble d'essais «Efficacité technique, efficacité sociale » avec son sous-titre «Le technique est-il dans le social ou face à lui ? » ne semble poser que deux questions: une première sur la notion d'efficacité sociale, et la possibilité de l'assimiler à l'efficacité technique; une seconde sur l'autonomie du geste technique par rapport au comportement social. Cette apparence est pourtant trompeuse, du fait déjà que les deux compléments circonstanciels de lieu qui apparaissent dans la deuxième question - «dans la société » et «face à la société »- n'ont pas le même poids sémantique en ce qui concerne l'étude à entreprendre.

2 Nous sommes donc dès le départ confrontés à trois thèmes : deux énoncés principaux dont l'un comporte deux énoncés subordonnés. L'ordre dans lequel on aborde ces thèmes est d'une importance primordiale. En effet, inverser cet ordre pour discuter d'abord de l'autonomie, puis de l'efficacité, peut transformer immédiatement, sans raisonnement préalable, donc sans justification, les hypothèses d'une problématique en pseudo-résultats d'une recherche... et vice versa.

Par exemple commencer la réflexion en se demandant si le technique est dans le social (première hypothèse du second énoncé) revient en quelque sorte à proposer que technique et social ne sont que deux faces d'une même réalité, et la discussion porte alors sur la possibilité d'appliquer sans ambivalence aux deux aspects la même notion d'efficacité. En revanche, commencer en supposant que le technique peut être face au social revient à adopter implicitement la position que le technique et le social sont autonomes. Il s'ensuit qu'il pourrait fort bien s'agir de deux types distincts de réalité. Naît alors la nécessité de discuter de la nature de la réalité, question épineuse s'il en fut $^{1}$. Par ailleurs, soutenir que ces réalités différentes sont face à face crée immédiatement un paradoxe, car l'approche fondamentale de toute étude technologique part $\mathrm{du}$ principe que, parmi les productions sociales, figurent les 
techniques. Et placer celles-ci délibérément à part - peu importe si c'est en face, à côté, en dessous, au-dessus- non seulement dévoile ce paradoxe, mais aussi ressemble étrangement à une pétition de principe. En revanche, la difficulté d'employer la même notion d'efficacité pour le social et le technique s'estompe en grande partie, car par avance les deux domaines ont été séparés.

Quant au premier énoncé, dans cet essai la notion d'efficacité sera réservée au domaine des techniques, et sera comprise dans son sens habituel, lequel est d'établir une relation mesurable entre ce qui entre dans une boîte noire et ce qui en sort. Pour ce qui est du domaine du social, j'ai argumenté ailleurs que tant les structures que les fonctionnements techniques peuvent forcer les barrières et les verrous.

5 Il faut bien dire qu'il semble que nous ayons élaboré un sujet d'étude d'une telle complexité, que le doux susurrement que l'on entend dans la distance signale assurément la nécessaire approche de la lame du rasoir d'Ockham.

Dans les lignes qui suivent, je vais tacher de garder aux questions toute leur complexité, quel que soit le niveau d'analyse, en vrai technologue me dira-t-on sans doute, avant non seulement de proposer quelques réponses et d'indiquer ce qui me semble être les indispensables directions de recherche, mais aussi de suggérer un cadre conceptuel pour l'étude des rapports entre techniques et société.

L'argument

7 Reprenons d'une façon plus formellement rigoureuse et donc, espérons-le, plus claire, l'architecture épistémologique de cette réflexion. La problématique d'une recherche de la ou des réponses doit donc comprendre les justifications de la question même, en sus des définitions préalables des termes et concepts auxquels on aura à faire appel, et dont ici la rigueur est réellement incontournable. Au risque de paraître bâtir un piédestal monumental pour une stature de moyenne taille, il va falloir justifier la position selon laquelle les techniques relèvent d'un domaine d'étude différent dans son essence du domaine des phénomènes sociologiques. Alors, mais seulement alors, pourra-t-on soulever d'abord la question de leur situation analogique et ensuite le problème de leur efficacité. Cet ordre est nécessaire, toute autre succession dans l'exposition des arguments nous mènerait forcément dans le «slough of despond $d^{2}$ » pour emprunter à John Bunyan, dans sa description du «Voyage du Pèlerin » une expression ô combien parlante pour décrire le lieu où se retrouvent souvent les chercheurs en anthropologie.

8 Le point de départ, en réalité, est un effort pour combler une fracture qui se découvre avec une apparence de fatalité chaque fois que les observateurs "pratiquants » des phénomènes relevant du domaine de la technologie culturelle rencontrent sur le terrain de la théorie les "penseurs" des relations sociales. Tout se passe comme si ceux-là démontraient à l'envi qu'une technique donnée est une production sociale, et que ceux-ci répliquaient en mettant quiconque au défi de prouver la pertinence des techniques pour l'analyse des entrelacs d'un système social ou a fortiori symbolique. L'économie peut-être, le geste technique certainement pas.

Quelle route prendre pour résoudre ces conflits, autre que celle qui permet de constater la futilité des formules à l'emporte-pièce? L'hypothèse de travail qui me paraît la plus potentiellement fructueuse est de postuler, premièrement, que les techniques peuvent éventuellement occuper un espace face aux phénomènes sociaux lorsqu'il s'agit de réfléchir sur les « domaines » réunissant les faits à analyser. Ensuite, le technique serait plutôt à côté du social quand il est question de la méthodologie 
d'approche et d'analyse de phénomènes particuliers. Enfin le technique est dans le social lors du traitement de la société globale.

Précisons le sens donné ici aux termes : « face à ", «à côté de ", « intriquée dans », sans pour autant verser dans un formalisme outrancier, car ces termes ne sont que des raccourcis pour des métaphores, auxquelles il faut se garder de donner une substance concrète. Le terme "face à » suggère la notion de conflit ou heurt entre les éléments des deux entités en question. Éventuellement il peut y avoir accord, mais de toute façon, pour ainsi dire, chacun reste sur son quant-à-soi. " À côte de " suggère la coopération, voire une certaine forme d'imbrication entre les éléments, mais le geste technique garde toujours son appartenance à un processus, un comportement social répond toujours à un paradigme distinct.

11 Ensuite, pour faciliter les efforts entrepris pour comprendre ce qui se passe ici-bas, il est possible de répartir les phénomènes observés en trois domaines: nature, techniques, société. Il est bien entendu que si la réflexion repart dans l'autre sens, vers des abstractions de plus en plus générales, force est de constater que tout appartient au domaine de la nature, les trois règnes (animal, végétal, minéral) classiques en ce qui concerne la planète terre, puis les espaces sidéraux et ainsi de suite. Maintenant, lorsque l'on considère en bloc des phénomènes dans une société globale, il est légitime de faire ressortir de façon globale une situation de conflit entre le technique et le social, ce qui est le cas pour les relations de face à face. Par ailleurs, les rapports de causalité entre deux unités face-à-face sont plutôt linéaires, au contraire des rapports passablement complexes de cause à effet entre deux unités juxtaposées. Que signifie « linéaire " ? Il s'agit là de rapports sans feed-back notable, ou alors d'une rétroaction diffuse et globale. Par exemple, l'introduction des tramways au Caire à la fin du XIXe siècle rencontra une forte opposition de la part des clercs musulmans en raison de l'impossibilité de trouver une référence précise dans le Coran à ce qui pourrait ressembler à un tram. Le technique et le social étaient alors en conflit. Le premier finit par avoir gain de cause, mais cet événement ponctuel n'a plus eu de suite, ce qui veut dire simplement que le technique et le social ne se sont plus réellement affrontés sur ce point précis. C'est ce que j'appellerai une relation linéaire.

12 En revanche, lorsqu'il est possible de mettre en relation l'ouverture des mines de borax en Californie, la construction des " clippers », le commerce de thé entre l'Angleterre et les Indes, l'invention des coiffures féminines "permanentes ", la ruée vers l'or, les conflits entre le Mexique et les États-Unis, comme le fait James Burke (1996), il s'agit des rapports complexes, non linéaires, en constant état d'interaction et de rétroaction. Il faudrait probablement aussi parler plutôt de rapports de renforcement, d'opposition ou d'indifférence que de causalité. Notons un aspect important de cette analyse, qui est l'indéniable vérité qu'aucune situation sociale n'est permanente. Au fil du temps, les relations du type " à côté de " peuvent se transformer en rapports de face-à-face.

Enfin, le concept de techniques « imbriquées dans le social " signifie que des décisions techniques sont prises pour des raisons sociales et vice versa. Prenons le cas où on ne modernise pas une opération métallurgique car cela entraînerait une mise en doute des relations unilatérales et univoques d'autorité au sein d'une famille. Il s'agit en l'occurrence de la difficile, voire impossible introduction de la soudure à l'arc dans une forge traditionnelle marocaine. En effet, la connaissance technique de cette forme de soudure est détenue par le neveu du forgeron. L'arrivée d'un arc à souder mènerait le 
neveu à enseigner sa connaissance à son oncle. Ce rapport entre aîné et jeune, et, qui plus est, entre oncle et neveu, est inenvisageable dans la société traditionnelle.

Autre cas, au Maroc encore : un réservoir d'eau pour des canaux d'irrigation n'est pas bétonné parce que, selon les usagers, il incombe à l'État central de subvenir aux besoins de groupes locaux. Cette perception du rôle de l'État est liée aux notions traditionnelles de l'autorité politique, du haut vers le bas, du père vers les enfants, du vieux vers les jeunes. Le technique n'a que peu à voir dans cette situation, les populations locales sont parfaitement capables de bétonner un réservoir, ou d'accomplir bien d'autres gestes techniques. D'ailleurs, l'un des aspects les plus réjouissants du Maroc moderne est de voir les associations villageoises prendre la situation en main, amener l'électricité en créant des batteries solaires et des moulins à vent (Ali Amahan 1999). Notons en passant, en ce qui concerne la recherche, que ce type de fait social total est difficile à traiter autrement que banalement lorsqu'il s'agit d'unités globales. Nous avons une recherche en profondeur à faire pour créer un mode d'analyse qui évite de perdre de l'information en allant du fait vers l'abstraction. On peut donc déclarer à juste titre que tout est imbriqué au sein d'une société; mais pour celui qui veut étudier les rapports entre différentes manifestations de la vie sociale, il faut auparavant opérer des distinctions et des catégorisations méthodologiques.

Les outils conceptuels qui aideront à faire comprendre ces hypothèses sont d'une part la chaîne opératoire et d'autre part les niveaux d'observation et d'analyse. En d'autres termes, la position théorique adoptée qui donne quelques chances de sortir de l'imbroglio créé par le l'intitulé de la réflexion, lequel pose, certes, des questions pertinentes, mais se fonde aussi malheureusement sur des concepts dogmatiques et réitère des idées reçues - parfois mal digérées-, cette position s'énonce comme suit. À certains moments et en certains sites du paradigme du fonctionnement social, le geste technique est largement autonome par rapport aux institutions et le chercheur assure l'ancrage de ce geste dans la société en « écrivant » les gestes d'un processus technique et les rapports sociaux activés par ces gestes dans une même "équation " (Cresswell 1994). Le technique est ici à côté du social, mais n'importe quelle technique n'est pas à côté de n'importe quel social. La chaîne opératoire n'est ni gratuite -libre à chaque chercheur de l'agencer comme il l'entend-, ni neutre -sans signification réelle. Cela n'empêche pas de soutenir que, dans ce qu'ils ont d'essentiel, le domaine technique est autonome par rapport au domaine social, et que celui-là est médiateur entre nature et culture. À d'autres moments et à d'autres niveaux, les processus et les produits techniques sont intimement intriqués à chaque instant du fonctionnement social et donc, ils doivent l'être aussi à chaque instant de l'analyse, quelles que soient les méthodes de recherche utilisées, ce qui appelle l'emploi du paradigme des niveaux, abordé à la fin de cet essai.

Revenons à la traduction graphique d'une chaîne opératoire qui permet de codifier les relations entre gestes techniques et comportements sociaux. Son utilité dérive de ce qu'elle décrit des processus et des structures, et ne consiste pas dans des juxtapositions d'analogies morphologiques. Il faut bien entendu éviter les tentations ludiques de ce genre d'exercice, notamment celles engendrées par l'usage de tableaux et de rapports sous forme d'équations, tentations auxquelles bon nombre de disciples de l'école structuraliste ont cédé au grand dam de l'intérêt de leurs conclusions. Le garde-fou du graphique ici proposé, auquel nous viendrons dans un instant, est qu'il est ancré dans du concret, le social et le technique s'écrivant avec les mêmes termes. Le processus de 
fabrication d'une houe -où d'une Ferrari-met en œuvre une séquence de gestes dont «l'ordre » est fixe. On ne trempe pas l'acier avant de l'avoir chauffé, on ne pose pas le carter d'un moteur avant d'avoir fixé les bielles. Partant, les attaches sociales de ces gestes apparaissent dans une progression fixe.

Ensuite, il sera question du développement de ce graphique, rendu nécessaire par les mutations des dernières années dues à la transformation des composantes de la population active, qui a modifié la nature des rapports dialectiques entre technique et société. À l'avenir, les recherches en technologie culturelle devront être orientées vers une meilleure intégration des produits et des intentions des acteurs dans l'étude des processus, et des rapports de ceux-ci avec les structures. Il s'agit d'une réelle nécessité. Les techniques dites traditionnelles, qu'elles se pratiquent de nos jours ou datent des ères plus anciennes, présentent un trait en commun, celui d'être l'activité principale, sinon de la majeure partie, au moins d'une grande proportion de la population. De ce fait, elles entretiennent des rapports plutôt linéaires avec les structures sociales en lesquelles elles sont intimement imbriquées. En revanche, les techniques actuelles, notamment celles qui relèvent de la " haute technologie ", sont des activités qui ne sont plus pratiquées que par une petite minorité de la population au travail ${ }^{3}$. Cela implique, entre autres conséquences, que les produits des techniques ne doivent plus être étudiés comme des " objets " à analyser à part en tant que témoins sociaux ou psychologiques de la société ou de l'individu, mais comme des facteurs intrinsèques des processus techno-sociaux.

Enfin, il faudra examiner l'hypothèse qui énonce que la seule manière de concevoir et donc d'analyser les rapports entre technique et société est de les concevoir comme faisant partie intégrante d'un "réseau sans coutures", a seamless web. Cet énoncé représente un effort pour résoudre d'apparentes contradictions dont pourtant les termes sont fondées sur des observations de terrain on ne peut plus fiables, et en outre parfaitement justifiables par une logique établie à partir de longues réflexions d'anthropologues qui sont, quant à eux, non moins fiables. L'un des résultats, sans doute non voulu, de cette manière d'envisager les phénomènes est d'émietter les gestes techniques à tel point que toute structure technique risque de devenir invisible, voire introuvable, de sorte que le domaine d'étude est placé hors d'atteinte raisonnable.

Notons en passant que ce point de vue conforte l'idée que tout est relatif, ce qui amène certains raisonneurs de l'avenir à postuler que rien n'existe en dehors de la conscience humaine, jusques et y compris les lois physiques. L'outil conceptuel qui aidera à situer la place réelle de ce concept de web dans l'étude sera celui des niveaux d'observation et d'analyse. Enfin, je vais encore postuler que les contraintes matérielles agissent sur les processus techniques de façon à rendre ceux-ci tributaires de celles-là, mais de façon tout aussi péremptoire, je poserai que les structures sociales encadrent les processus techniques pour empêcher (il s'agit encore de "verrous ») ou favoriser l'innovation, voire la régression.

20 En aparté, il me semble bon de faire remarquer qu'à l'évidence, en tant qu'être social, tout chercheur peut justement prétendre avoir une connaissance pratique des relations sociales puisqu'il les vit lui-même. Ipso facto les jugements qu'il porte sur la justesse de son analyse, ou sur la pertinence des constructions intellectuelles d'autres chercheurs, sont fondés en partie sur un vécu. Son propre apprentissage des rapports fut acquis "sur le tas ». En revanche, les connaissances dont dispose ce même chercheur quant aux gestes techniques en général ou en particulier sont rarement autres 
qu'intellectuelles; cette faille dans le vécu ou dans la pratique atténue sérieusement la portée ou la pertinence de ses analyses. Viser ces buts dévoile donc trois grands problèmes : comment inclure dans la description et l'analyse les intentions des acteurs -d'autant que celles-ci naissent souvent dans le domaine du symbolique social et culturel; comment traiter le problème soulevé par le concept du "seamless web" souvent invoqué par les chercheurs dans ce domaine de la technologie culturelle; et enfin, comment tenir compte de l'évolution des techniques, en un mot de l'histoire?

Discussion

Commençons par une réflexion sur les méthodes de recherche, et abordons accessoirement le problème des paradoxes. L'une des premières pierres d'achoppement est la définition de l'efficacité. Quelle efficacité, comment la mesure-t-on ? Y en a-t-il deux sortes, technique et sociale, et en fin de compte que signifie " efficacité » ? Et puis poser ce genre de question ouvre potentiellement la porte à un raisonnement sans fin. Par exemple, un sociologue ou un anthropologue étudie le système de la parenté dans une société déterminée. Or, il appartient lui-même à un groupe social et partant, il devient important pour son analyse de l'autre de savoir comment sa propre appartenance, son vécu influence les modèles qu'il crée. Ensuite, l'observateur critique qui réfléchit sur ce sociologue et sa sociologie est aussi un être social et son regard est loin d'être neutre. Où doit-on arrêter le cercle infernal de la sociologie de la sociologie de la sociologie ad infinitum ? Encore une fois, débarrassons-nous des entités superflues.

Alors comment faire pour trouver et pour saisir l'extrémité d'un fil d'Ariane, si tant est qu'il existe, où trouver les ciseaux pour le couper au moment opportun? Tout d'abord en convenant d'une série de définitions, sans trop se préoccuper du choix du meilleur terme. Dans ce cas, la nomenclature n'est pas d'importance primordiale. Un travail anthropologique sur les rapports entre techniques et société appelle à définir technique, société, et culture comme objets d'étude, et comme sujets pour l'analyse, à délimiter les champs et sous-champs de la technologie, de la sociologie et de l'anthropologie.

On peut se demander pourquoi il est nécessaire de définir le technique. Tout simplement parce qu'il est fait un usage tellement général de ce terme qu'il faut tracer des limites du domaine. Est technique tout procédé de manipulation par l'homme d'un matériau naturel en vue de le transformer en produit ou objet utilisable. Bien entendu il peut s'agir d'une manipulation de produit déjà transformé. Il faut donc un agent, un outil et un matériau pour qu'un geste technique puisse avoir lieu, pour qu'un processus technique soit activé. À l'extrême limite existent des techniques du corps, là où agent, outil et matériau se confondent en un seul lieu, le corps humain. Mais il ne saurait être question de «techniques» dans d'autres domaines, politiques, artistiques, psychologiques que sais-je ?, où le mot est pris dans le sens d'une métaphore.

Dans le cadre de cet article, on considérera comme société un groupe de personnes qui ont conscience de leur identité collective et de leur spécificité et qui se sont donné consciemment des règles et des institutions pour gérer leur comportement individuel ou collectif.

On appellera "culture " la configuration particulière qu'adopte chaque société humaine non seulement pour régler les rapports entre les faits techno-économiques (y compris l'interaction avec le milieu naturel), l'organisation sociale et les idéologies, mais aussi pour transmettre ses connaissances de génération en génération. Gestes techniques, comportements sociaux (individuels ou collectifs), institutions juridiques et 
politiques, systèmes symboliques, savoirs et modes de transmission des connaissances et des comportements dans chacun de ces domaines figurent donc parmi les éléments les plus importants d'une culture spécifique. Ces différentes activités se constituent en réseaux et chaque culture se distingue par une manière particulière de structurer ces réseaux et de les articuler entre eux. Culture et société ne sont pas synonymes. Une culture peut recouvrir plusieurs sociétés, de même qu'une société peut abriter plusieurs cultures. Selon que l'un ou l'autre des réseaux est privilégié comme sujet d'analyse, l'aspect et l'échelle de la configuration changent. Par exemple, une culture « industrielle » engloberait et la Russie et les États-Unis d'Amérique, bien qu'à un autre niveau de perception, il existe une culture américaine et une culture russe. Il est immédiatement évident que l'un des avantages de cette définition est de ne pas figer les cultures ou les sociétés dans des limites strictement tracées. Cette liberté épistémologique permet de traiter le dynamisme des cultures, les processus d'innovation sociale ou technique, le déplacement de signification des symboles, les zones d'interaction entre réseaux, et souligne la nécessité de rechercher les structures profondes, toutes choses difficiles sinon impossibles à analyser à partir de structures rigides, gelées dans un moment ponctuel de l'histoire.

L'ampleur de cette définition de la culture par rapport à celle de la société ne tient pas uniquement à ce qu'il s'agit dans le premier cas d'un concept qui fut élaboré par la discipline universitaire de la sociologie, et dans le second par celle de l'anthropologie loin de moi toute tentation de m'enfermer dans des cadres formels et surtout arbitraires-, mais à ce qu'en termes d'appréhension, la société est un objet perçu, descriptible en termes de tangibilité, tandis que la culture est un concept, un sujet d'analyse. Ce qui ne signifie nullement que la société ne soit jamais un sujet non tangible d'analyse -il n'est que de penser aux symboles de préséance-, ni que la culture ne s'exprime jamais de façon tangible -il n'y a qu'à réfléchir sur les symboles artistiques-, mais simplement que société et culture se situent à deux niveaux d'analyse différents.

Passons aux domaines de recherche et aux sujets d'étude. En premier lieu le terme « technologie » est posé comme l'étude des techniques; dans le domaine industriel, par exemple, l'analyse du fonctionnement d'une machine-outil, disons, ou d'une chaîne de montage robotisée et informatisée, consistera en la description des séries de gestes techniques -coupe, rivetage, soudure, perçage, assemblage, peinture, étiquetage...-, en une succession de tâches, en l'identification des flux, en la détermination et la mesure des forces mises en jeu, en l'élaboration de chemins critiques, etc. Tous ces facteurs et vecteurs peuvent être décrits - je dis bien "décrits »- sans référence à la société. En revanche, pour les analyser, pour étudier leur développement par rapport à la science dite dure sinon humaine, en un mot pour essayer de comprendre leur enchâssement dans le social, il faut faire largement appel à la société dont ils sont une des expressions. À partir de ce changement nous parlerons de «technologie culturelle ».

De même, les sciences sociales étant l'étude des relations entre humains, la description des relations de parenté ou du fonctionnement d'un système politique ou de n'importe quelle institution qui cristallise ou qui régule ces relations peut éventuellement se faire sans entrer dans les détails techniques de la construction d'une mairie ou d'un parlement. 
29 Il reste à souligner un aspect de l'anthropologie que je trouve essentiel. En évitant les polémiques, posons simplement que l'anthropologue a peut-être à l'esprit un souci holiste plus constant que n'a le sociologue.

Nous arrivons maintenant directement au cœur de la problématique du présent essai. Si déjà nous séparons techniques et société au niveau des définitions, ne préjugeonsnous pas de la réponse aux questions posées? Voici une première approximation de l'approche de ce problème fondamental. Quand bien même on affirmerait qu'une technique est une production sociale, qui ne peut s'analyser sans se référer à la société, il reste à déterminer la métaphore adéquate : s'agit-t-il d'une gangue dans laquelle la technique est sertie, ce qui présuppose l'existence autonome et de la technique et de la gangue, ou s'agit-il d'un tissu sans coutures, d'un continuum sans rupture? Cette question rappelle le conflit qui séparait les deux écoles principales de physiciens dans les discussions sur les équations devant unifier les quatre forces fondamentales (gravitation, électromagnétique, «forte », et « faible») dans une TDT (théorie du tout), l'affirmation ou le rejet de telle ou telle théorie relevant de deux visions opposées de la nature de l'univers: celui-ci est-il une substance unique comme la gélatine, ou est-il composé de particules discrètes? Si j'évoque ces discussions, ce n'est pas pour chercher à tout prix des analogies, mais simplement pour faire remarquer qu'un différend en ce qui concerne la TDT n'empêcha pas l'humanité de manipuler les aspects tangibles de cet univers, que ce soit en envoyant des hommes sur la lune ou en fabriquant des microscopes pour photographier des molécules.

Cela posé, il faut éviter un rets tendu par cette idée que techniques et société tissent un seamless web, car autant ce concept donne bien une vue globale pertinente, une intuition éclairante pour ce qui est de la compréhension du cadre général, autant il devient dirimant lorsque le champ se développe jusqu'à inclure des détails techniques ou sociologiques. La logique qui sous-tend la contradiction est implacable. Il est évident que tout phénomène social, si l'on parcourt les chemins de tous ses liens de rapport en rapport -sans se préoccuper de l'importance relative de chaque relation-, est lié à chaque autre phénomène dans une société donnée, qu'il s'agisse de processus ou de structure, de geste technique ou de rapport social. Mais il est non moins évident que si l'on étudie une série de gestes techniques, en un mot un processus dont les liens entre gestes sont nécessaires, où l'ordre dans lequel se succèdent les actions dans les séquences est aussi important, sinon plus, que le geste même, l'architecture du réseau technique oppose une forte résistance aux changements, et peut éventuellement s'imposer aux phénomènes sociaux dans le sens que les attaches sociales de différents gestes ne se lient pas forcément entre elles pour former un réseau social qui correspond terme pour terme au réseau technique. J'entends par là que deux gestes dans un processus technique présentent une forte interrelation. Chaque geste comporte une connexion sociale immédiate, mais ces deux connexions ne montrent pas le même rapport «fort ». Par exemple, les rapports techniques entre deux étapes dans la fabrication d'une automobile constituent un processus fortement cohérent (fig. 2). Il n'en va pas de même en ce qui concerne les attaches économiques de l'étape $\mathrm{x}$ (emboutissage d'une porte) et les institutions comme la jurisprudence attachées à l'étape y (vente). Ces attaches-là sont lâches ou plus faibles que les liens techniques.

or, lorsque deux analyses - seamless web contre face-à-face- sont contradictoires, que fait le chercheur? Il révise la logique qui sous-tend son analyse. J'ai rencontré ce problème au Liban. La définition de structure que l'on m'avait enseignée à l'université 
aussi bien à Princeton avant la guerre de 1939-1945 qu'à la Sorbonne ensuite, ne s'accommodait pas des faits observés sur le terrain. Dans pareil cas, c'est aux définitions de s'expliquer, ce n'est pas au terrain de s'y conformer. La réponse à ce problème particulier menait à postuler une autre notion de structure qui prévalait au Moyen-Orient (Cresswell 1966). Pour le dire brièvement, la notion de structure occidentale correspond à une architecture de hiérarchies. La notion de structure au Moyen-Orient est celle d'un système de contrats qui sont à renégocier à chaque changement dans le statut et le rôle des partenaires.

Dans le cas qui nous préoccupe ici, pour répondre à la première question, posons l'hypothèse que les techniques sont à la fois dans la société et face à la société et que le choix entre les deux arrangements se fait selon le niveau d'analyse où on se place. À ce propos, notons en passant une attitude curieuse. Les études de technologie culturelle disent bien que le technique ne se comprend pas sans prendre en considération le social, mais je connais peu d'analyses qui inversent la proposition : le social ne livre pas ses secrets sans inclure le technique.

La vue du seamless web prise depuis le sommet de la montagne donne par conséquent une perspective qui situe de façon juste les éléments du paysage les uns par rapport aux autres. Cette perspective permet l'expression d'analogies évocatrices, certes, mais potentiellement trompeuses quant à l'analyse fine. «Une mer de nuages » est une expression courante, jusque dans les médias, qui fait naître dans l'esprit de l'interlocuteur -tout au moins s'il a déjà vu la mer- sinon une sensation poétique, au moins une image juste de ce que voit l'observateur qui lui parle. Mais le développement de cette sensation, l'extension du vécu, n'est d'aucune utilité pour l'étude de la formation de gouttelettes de $\mathrm{H}_{2} \mathrm{O}$ dans les gaz de l'atmosphère terrestre. L'intuition n'est pas -pas encore- une méthode d'étude opératoire ${ }^{4}$. De même, la répartition des phénomènes observés dans les champs disciplinaires : physique pour les forces, chimie pour les relations moléculaires, voire biologie pour les fonctions organiques, et cela sans parler de la tendance actuelle, tout à fait louable, à chercher de nouvelles combinaisons de méthodes afin de mieux comprendre, n'est d'aucune utilité pour l'étude détaillée. De fait, cette nouvelle approche ne fait qu'ajouter un autre niveau sans en détruire les précédents.

Nous posons alors explicitement qu'il est légitime de déchirer le tissu pour séparer le domaine technologique du domaine sociologique (s'agit-il d'une différence de nature, voire d'une distinction épistémologique, ou s'agit-il d'une astuce d'écriture pour contourner les difficultés de l'exposition linéaire ?). Ajoutons qu'il faut veiller à ne pas tomber dans le piège que n'ont pas su éviter ceux qui, sous prétexte qu'il est question de travail, confondent techniques et économie, c'est-à-dire qui, au lieu de tenter d'ouvrir une boîte noire, en créent une autre. Il s'agit bien du technique stricto sensu. Cela n'empêche pas de reconnaitre en quoi le terme seamless web rend parfaitement compte de l'une des caractéristiques des rapports entre technique et social. Soulignons aussi que la compréhension de la manière dont les techniques contribuent au modelage de la dimension sociale de l'homme reste une image opaque tant que n'est pas révélée simultanément l'image symétrique : comment l'intervention de la société détermine-telle la direction de l'évolution des techniques?

Néanmoins, en dehors de cette hypothèse de travail, il existe une justification réelle pour séparer geste technique et comportement social dans l'analyse même. Car autant il est légitime de parler de progrès ou régression dans les processus, produits ou 
systèmes techniques, autant il est interdit de le faire dans le domaine sociologique. De par son essence même, il est possible de qualifier une technique par son efficacité dans l'atteinte d'un but. Une plus grande efficacité d'une innovation technique marque un progrès, une moins grande, une régression. Il ne s'agit évidemment d'appliquer les termes efficacité et progrès qu'à des états physico-chimiques.

Mais c'est précisément ce caractère physique des techniques qui justifie notre démarche épistémologique. Pendant sa longue évolution l'homme a acquis des procédés pour manipuler et modeler le monde matériel à son avantage. Or, cette manipulation, comme dans une certaine mesure sa propre existence physique, sont des exceptions locales à la seconde loi de la thermodynamique. Selon cette loi, à la fin des temps, toute la matière de l'univers sera à la même température, de telle sorte que toute source potentielle d'énergie aura disparu, ainsi que la vie en conséquence. Ce mouvement -cette flèche irréversible du temps- est appelé l'entropie positive. La technique, activité essentiellement humaine, est un essai, jusqu'ici réussi, pour contrer la nature. Sur son existence physique, l'homme n'a -jusqu'à présent- que peu de contrôle, mais en ce qui concerne son rapport au monde matériel, on peut dire que la technique est un procédé humain pour aller en sens inverse du mouvement général de l'univers, pour créer de l'ordre là où la nature évolue vers le désordre. En quelque sorte, l'homme technicien réussit à forcer tous les haricots à sauter dans la même boîte. Cela est une des raisons pour lesquelles l'efficacité technique est payante : il s'agit d'un antidote local aux problèmes causés par une entropie positive générale. Le progrès, pour une technique, consiste en un usage toujours plus économe de l'énergie, et cette manipulation des forces naturelles peut être mesurée. Cette propriété des techniques, ce caractère intrinsèque, est essentielle pour leur compréhension. Le sens général de l'évolution des techniques depuis le premier biface jusqu'à l'industrie nucléaire tend vers un contrôle chaque fois plus complet du milieu naturel, vers une libération des contraintes imposées par les forces physiques, chimiques et biologiques de ce même milieu $^{5}$.

Évidemment, jusqu'à ce jour, une libération de certaines contraintes physiques par la technique a créé des contraintes certaines dans d'autres domaines, surtout dans le domaine social. Ne serait-ce que dans la centralisation étatique que semble exiger une forte industrialisation. Mais dire que l'évolution technique est porteuse de la notion de progrès, ce n'est pas affirmer l'inexistence de la dégénérescence technique -trop d'exemples historiques sont là pour prouver le contraire. Le retour en arrière peut être causé par un acte délibéré, tel que la destruction du système d'irrigation dans la steppe d'Iraq au XIIe siècle par les Mongols et la désertification persistante jusqu'à nos jours qui en a résulté. Il peut être aussi le fait d'un nivellement par le bas d'une technique avancée introduite dans un milieu technique plus attardé, ou plus exactement à un niveau de développement différent.

Quoi qu'il en soit, il importe de bien souligner que ce jugement de valeur sur les gestes techniques est autorisé au niveau le plus concret du fait technique. Exposer à côté du chopper est africain d'il y a 3 millions d'années l'un des Spoutnik ou des Apollo suffit pour interdire le doute quant à la direction de cette évolution. Des institutions sociales, en revanche, ne peuvent être qualifiées en tant que telles par des termes comme " progrès » ou « efficacité », que dans la mesure où l'on cherche à caractériser leur plus ou moins bonne adaptation à un état donné de manipulation technique de l'environnement. Ou dans la mesure où l'on veut souligner un plus ou moins bon 
ajustement entre les intentions du groupe qui les a élaborées et l'emploi que l'on en fait. Posons donc qu'il est légitime de séparer, en vue de l'analyse, les domaines d'activité de l'homme en phénomènes sociaux et phénomènes techniques sans qu'il s'agisse d'un artifice de la pensée. Aussi imbriqués l'un dans l'autre que l'huile et l'eau d'une solution hautement émulsifiée, aussi nécessaires l'un à l'autre que l'espace et le temps (sans le temps l'espace n'existe pas et vice versa), le monde technique et le monde social sont néanmoins des entités réelles.

Approfondissons encore cette différence radicale de nature entre technique et social. Les phénomènes techniques sont cumulatifs, linéaires, et ils ont tendance à se développer. Les phénomènes sociaux sont non cumulatifs, ponctuels en ce sens que le progrès ne peut être qu'un trait au deuxième degré, et ils ont tendance au conservatisme. Les techniques sont cumulatives en ce que tout progrès, toute transformation, tout remplacement d'une technique par une autre se fait par l'incorporation d'un savoir ancien. Le traitement de l'acier à haute température incorpore les anciens savoirs de traitement de l'acier ou du fer à basse température. La manipulation génétique des plantes incorpore les savoirs de simples transplantations. Le rouet, véritable invention si jamais il en fut, associe deux savoirs déjà acquis : l'emploi de la bielle et les techniques de filage - dont l'alignement et le tordage des fibres végétales pour en faire des fils. Les techniques sont linéaires en ce sens qu'il faut toujours un certain ordre de développement. On ne commence pas dans la métallurgie des métaux ferreux avec de l'acier, mais avec du fer fondu. Dire qu'il faut un certain ordre n'implique pas que cet ordre soit le même dans chaque région du monde. En Occident, on est passé du fer fondu à haute teneur en carbone au fer forgé par le processus de puddlage, avant de refondre les blooms dans le minerai de fer pour aboutir par le processus Siemens de co-fusion à la matière première de l'acier. En Chine en revanche, en partie à cause de la haute teneur en phosphore du fer naturel, les forgerons ont pu passer directement du fer fondu à l'acier en utilisant le procédé Bessemer de décarburation.

41 Cette distinction est importante, voire essentielle, pour qui veut rendre scientifique l'étude de l'homme et de ses œuvres. Prenons les classements de l'histoire naturelle comme illustration. Les espèces, les races et les variétés sont formées d'individus séparés les uns des autres de façon visible. Je dévale une pente dans les Rocheuses sur un animal que je sais être un cheval à la poursuite d'un autre animal que je sais être une stupide vache qui ne veut pas rester dans le troupeau. Trois espèces sont en action dans une relation momentanée. En revanche, si j'envisage de partir à la chasse d'un bovidé, je ferais bien d'y réfléchir à deux fois car je ne verrai jamais un bovidé parmi les troupeaux d'antilopes, de yaks, de gazelles ou de chèvres, encore moins des céphalophinés ou des hippotraginés. Toutes les catégories supra-spécifiques sont affaires de savants, et peuvent être transformées sans provoquer de changement chez les individus concernés. Il en est ainsi de la différence entre gestes techniques et comportements sociaux. Au contraire des premiers, les seconds sont présents quel que soit le niveau d'analyse. Notons enfin que bien des éléments de la culture, telle que nous l'avons définie, ne se rencontrent que sur le plan des abstractions analytiques. Laissons là cette esquisse d'une réflexion qui reste à poursuivre.

La chaîne opératoire

42 Ces principes généraux étant posés, passons au concret. Le premier pas sur ce chemin est de définir explicitement une chaîne opératoire, outil pour analyser les rapports 
entre le technique et le social (fig. 1). Comme on peut le constater, chaque étape d'une chaîne consiste en un certain nombre de descripteurs, d'éléments, d'actions, et en un résultat. Les descripteurs sont au nombre de quatre : un terme scientifique et un terme indigène, un lieu et un temps. Les deux noms sont nécessaires, car une opération qui paraît pouvoir se subdiviser en deux parties distinctes d'après une taxinomie-mode de connaissance peut très bien être perçue par l'utilisateur ou l'agent impliqué dans la chaîne comme unique d'après une taxinomie-mode d'utilisation. Par exemple, dans la chaîne opératoire de la préparation d'une peau de mammifère marin sur la côte ouest $\mathrm{du}$ Groenland, les Esquimaux donnent un seul nom, erkorpa, au procédé d'assouplissement, qui consiste à immerger la peau dans de l'eau douce avec du savon puis à opérer plusieurs rinçages (Le Mouel 1978). L'effet sur la matière d'une immersion avec du savon dans un bac n'étant pas le même qu'une immersion simple dans de l'eau pure, le mode de connaissance est parfaitement justifié à y voir deux opérations et à lui donner deux noms : lavage et rinçage. Le descripteur de lieu s'explicite de lui-même, quant au temps, il s'agit aussi bien de la date et de l'heure de l'opération, que de sa durée. Les éléments impliqués dans une étape sont d'une part l'agent et l'énergie qu'il utilise, d'autre part le ou les outils et la ou les matières premières. L'agent peut être humain ou animal; l'énergie peut être d'origine humaine, animale ou autre (air, eau, minéraux, végétaux...). Toutes les combinaisons sont envisageables, un homme, des hommes, un homme et un animal, des hommes et un animal, un homme et des animaux, une machine et une source d'énergie... L'outil comprend aussi bien les outils manuels, qui peuvent être actifs (marteaux) ou passifs (enclumes), les machines peuvent être simples (une seule opération) ou complexes (plusieurs opérations à la fois). La matière première est soit le matériau qui doit être transformé en produit, soit un matériau secondaire adjoint à une étape spécifique, soit éventuellement un produit provenant d'une autre chaîne.

Pour réaliser un projet ${ }^{\mathrm{a}}$ une relation est activée $\mathrm{e}^{\mathrm{b}}$ qui se transforme en une action sur un matériau et résulte en un produit. La chaîne se constitue d'un certain nombre d'étapes. Chaque étape comporte 4 descripteurs : nom scientifique, nom indigène, temps, lieu; et 7 éléments : agent(s) (humain(s), animal (animaux), moteur(s)), outil(s) (actif, passif, machine...), dont l'assemblage (l'activation de la relation) produit par le truchement d'une connaissance ou savoir-faire (la flèche) un geste technique et une action sur la matière (percussion, pression, assemblage...), qui, agissant sur une matière première, ou sur un produit déjà élaboré, ou les deux à la fois, résulte en un produit (outil, machine, objet de consommation...). 


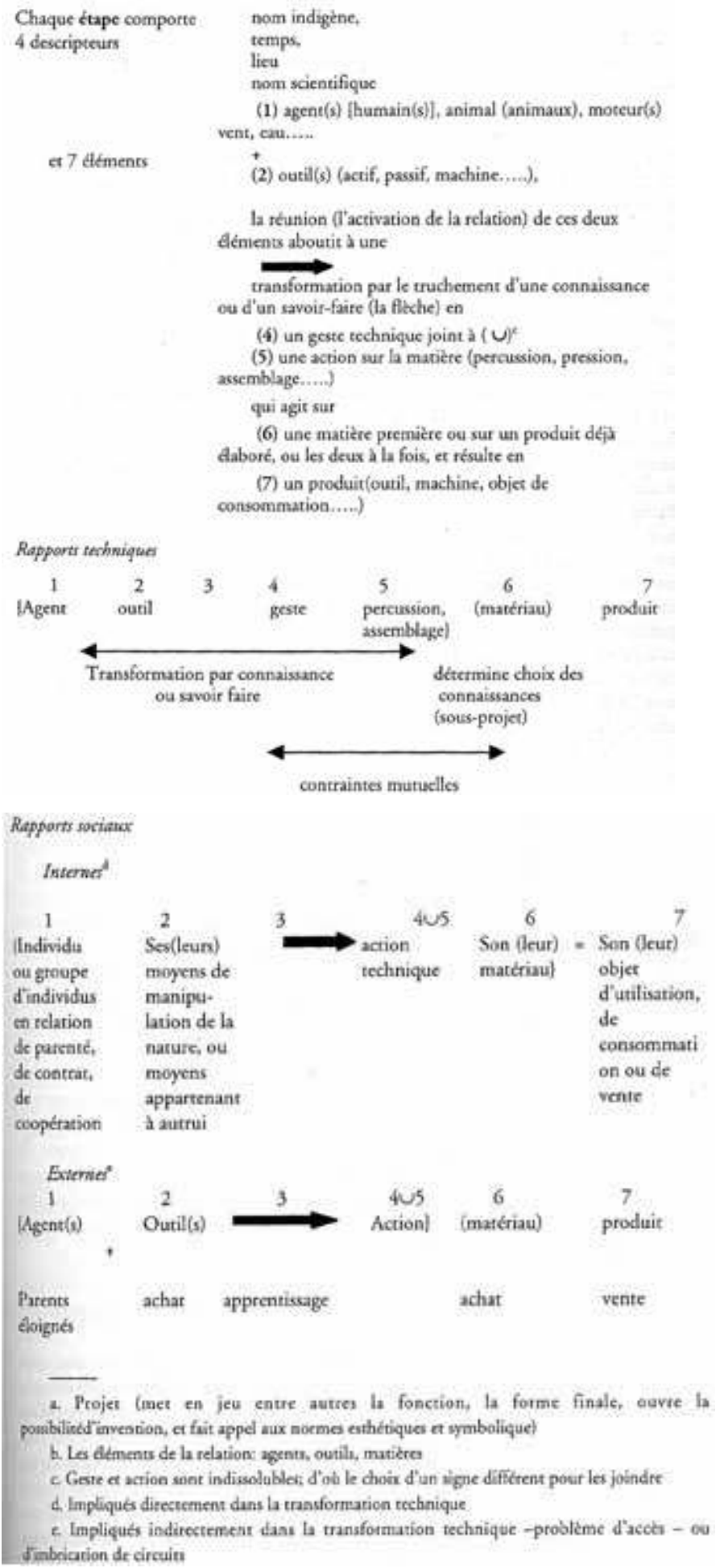

\section{Figure 1}

Commentaire

La chaîne opératoire, dont la principale vertu est d'intégrer dans un graphique unique et les gestes techniques et les structures sociales, permet de « coller » la réalité perçue (les rapports sociaux, le comportement relationnel des individus...) à la réalité objective (les séquences de transformation volontaire des matières premières), de même, 
puisqu'il s'agit d'un processus, donc d'un mouvement, est-elle capable de transcrire la dynamique de l'histoire.

Cette approche, et il faut insister sur ce point, n'est nullement limitée aux techniques traditionnelles. Le technicien qui appuie sur la touche de l'ordinateur et met en marche la machine pour emboutir une pièce de fuselage d'un avion en construction active par ce geste une relation qui est aussi bien sociale que technique, entre lui, son supérieur hiérarchique, le bureau d'études de la société qui paie le salaire lui permettant de s'abriter et de se nourrir, le comité d'entreprise et la direction générale, voire la société globale et ses institutions. Il ne fait pas autre chose que le forgeron marocain qui frappe sur une lame de houe traditionnelle tenue sur l'enclume par le frère de son père. Bien entendu, il reste à déterminer si le changement d'échelle transforme la nature profonde -l'essence- du geste, en un mot, si les différentes étapes entre le moderne et le traditionnel dans le domaine de la métallurgie comporte des solutions de continuité ou non.

Il faut maintenant mettre à l'épreuve cette graphie de la chaîne opératoire, non pas comme concept -après tout, il s'agit de relations indépendamment observables, voire mesurables, entre acteurs biologiques et objets physiques, donc réelles- mais en tant que potentiellement révélatrice des intentions de ces agents et acteurs. Et ce, forcément de façon à rendre possible la notation des différences entre les intentions des particuliers et ce qui est l'expression des tendances collectives. Cela, évidemment, pour pouvoir atteindre les structures et processus profonds.

La recherche technologique et le holisme anthropologique

Il faut que les outils de recherche et d'analyse permettent de tendre non seulement vers une théorisation en général, mais, plus important encore et cela pour plusieurs raisons. Tout d'abord, les sciences sociales, et plus particulièrement l'anthropologie pour des raisons qui relèvent des conditions de son développement, ne peuvent atteindre des explications plausibles que si les phénomènes observés sont remis à leur place dans un cadre général. Toute approche doit donc se situer dans une structure -le choix de ce terme est délibéré car il implique une architecture de relations. Par ailleurs, les limites de cette structure doivent dépasser de beaucoup celles du champ de recherche choisi. Pour emprunter une métaphore à Korzybski (2002), la carte doit être plus grande que le territoire, en un mot recouvrir un domaine plus vaste que celui qui est le sujet de la recherche.

Lorsque nous parlons de théorisation, il est important de savoir de quoi on parle. Il ne s'agit pas d'une analyse globale d'informations partielles, procédé qui, à la limite verse dans une sorte de nominalisme, le fait de nommer donnant exsistence à ce que l'on croit être perçu comme explication des relations entre phénomènes. Il s'agit de construire les conclusions d'une recherche de telle façon que les unités fondamentales à partir desquelles est bâtie une conclusion comprend toujours le réseau de leurs relations. (Chaque neurone vient impérativement avec son axone et ses dendrites.) Le but de cette exigence est de pouvoir replacer cet élément dns un autre réseau du domaine social, non pas simplement en juxtaposition aux autres éléments du domaine, mains intégré au nouveau système de relations. Une nouvelle pièce de tissu s'intègre dans un ancien morceau textile en passant les fils qui dépassent dans la chaîne et la trame de l'ancien.

D'où, soit dit en passant, l'intérêt d'« écrire " les relations entre gestes techniques et comportement social à partir des mêmes termes. Il n'est pas possible de trop souligner 
ici qu'il ne s'agit pas de tenter l'élaboration d'une TDT à chaque recherche, loin s'en faut. L'indispensable c'est que chaque pièce apportée à la mosaïque de l'ensemble soit taillée de façon à pouvoir y être insérée. L'analyse d'un comportement social doit permettre de le traduire en rapports économiques, en héritage d'un "patrimoine culturel ", en termes de parenté, en rapport avec les gestes techniques quant contenu substantiel; et de façon à permettre une expansion ou une abstraction sur le registe méthodologique.

Regardons un graphique passablement schématique de ce que pourraient être les chemins empruntés par un produit une fois terminé le processus de fabrication. Il s'agit en l'occurrence de la lame d'une houe provenant d'une forge marocaine d'une part, et de l'autre, d'une voiture moderne.

Nous voyons ici deux exemples du tracé des réseaux que peut emprunter un produit après le passage par une chaîne opératoire de production. Dans le premier cas, désormais classique, du façonnage d'une lame de houe traditionnelle, le produit passe directement à l'usager. Par la suite cette lame passera une ou plusieurs fois dans l'ensemble des chaînes opératoires; elle peut même retourner dans sa propre chaîne de fabrication pour réparation et, de ce fait, elle peut réactiver des réseaux socioculturels. L'important ici est que ce type de rapport entre acteurs sociaux et gestes techniques apparaît partagé par la très grande majorité des membres du groupe.

Dans le second cas, il s'agit de l'assemblage de pièces pour fabriquer une voiture automobile. Bien que cette production soit le résultat de l'interaction de dizaines de chaînes, sinon de centaines, ce qui implique l'activation d'autant de réseaux socioculturels, avec tout que cela peut comporter de verrous ou de renforcement des réseaux, il existe une différence essentielle entre la lame de métal et l'automobile. D'une part, la personne qui prend possession de l'automobile n'est impliquée ni dans le processus technique de fabrication, ni probablement dans aucun autre processus technique. D'autre part, le fait de ne pas participer aux techniques de son groupe social est la situation de trois-quarts des autres membres dudit groupe. Il est évident qu'il n'est pas envisageable de ne caractériser les manières d'agir d'un groupe que par les activités d'une petite minorité. On doit donc porter l'attention sur ce qui advient par la suite du produit technique. Il faut surtout que la diffusion du produit soit considérée tout autant comme partie intégrante du processus technique que sa fabrication, et que l'ensemble fabrication-diffusion soit autant un fait social total que chaque geste dans la chaîne. 


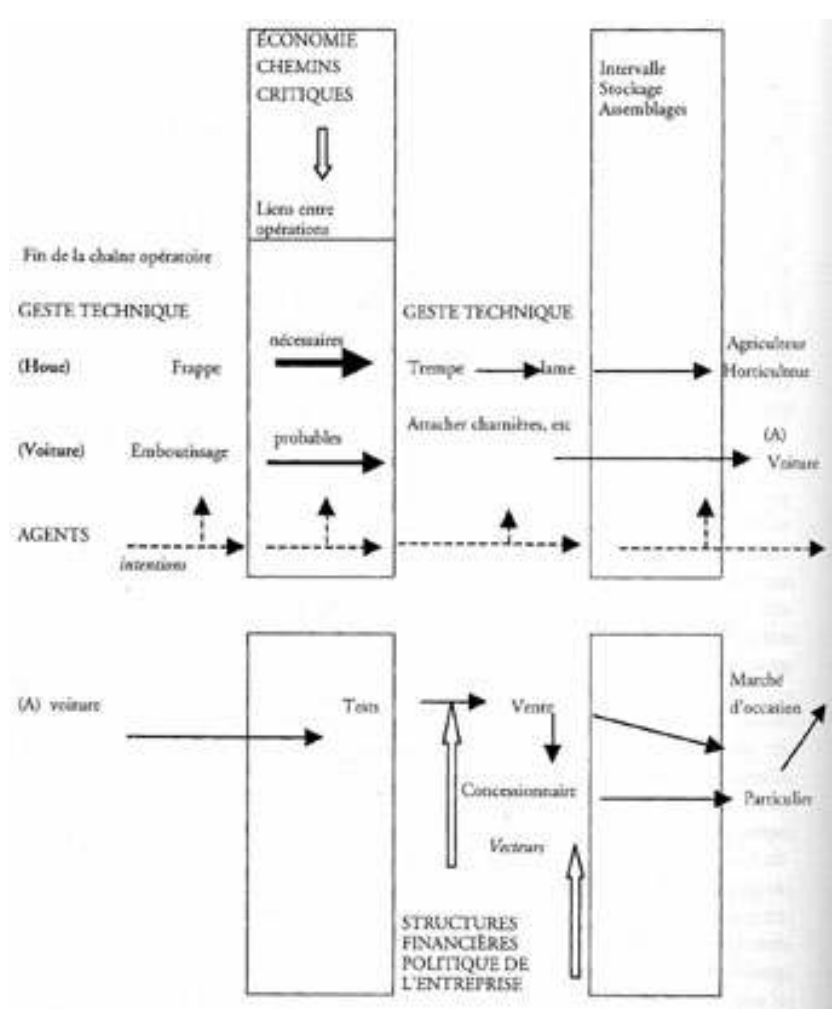
la politique, pour ne mentionner qu'eux, font de chaque geste un fait social total, mais la constante interaction des vecteurs appelle une étude à venir.

Les niveaux d'analyse comme cadre de la réalité techniques et les phénomènes socioculturels ne débouche pas encore sur une véritable résolution des contradictions posées en début de réflexion. Il faut toujours poser la question : s'agit-il de deux domaines face à face (éventuellement côte à côte) ou d'une intrication totale des techniques et de la culture dans un seamless web? Le dilemme paraitt toujours d'airain et sans issue. 
60 N'ayons pas recours à la violence, laissons Alexandre à Gordes. Élaborons un cadre qui représente la réalité de nos sociétés dans ce qu'il y a d'englobant, un ensemble de comportements qui apparait comme un tissu, chatoyant de par ses transformations constantes ou ternes de par sa structure en fils de chaîne. Saisissons cette entité globalement, tel que peut le faire le cerveau humain d'après les dernières études, comme un ensemble dont seules les contingences linguistiques nécessitent une linéarité du discours.

61 Ce cadre (fig. 3) comprendra trois colonnes principales et quatre rangs. Les rangs figurent les quatre niveaux de description et d'analyse: (1) les faits individuels, (2) les assemblages de ces faits, (3) les structures inconscientes, non perceptibles par les acteurs sociaux, mais culturellement spécifiques par rapport au niveau (4), où si situent les structures universelles, telles que les paradigmes mentaux de l'humanité et les formes ou la composition de la psyché humaine.

Les colonnes partagent les phénomènes entre les domaines de la nature, des techniques, et de la société.

63 Il reste à déterminer comment passer d'un niveau à un autre, d'un domaine à un autre, sans perdre dans cette translation les caractères intrinsèques de l'objet ou toutes les ramifications du sujet. Remarquons tout de suite, sans nous attarder, qu'il est évident que le terme de niveau n'est pas neutre car, entre autres, il implique une solution de continuité entre deux aspects d'un même phénomène. Il faut donc pallier les possibles effets néfastes de cette connotation implicite. Expliquons-nous. Soit un observateur venu d'ailleurs, qui voit en ma compagnie un groupe de gens, des deux sexes mais d'âges différents, en train de discuter dans une des pièces d'un bâtiment grand et imposant. Il note et décrit ce qu'il observe. Puis il remarque que je suis moi-même en train d'analyser en situation la structuration de l'espace, les vêtements, le langage, et les comportements. Il note que je semble comprendre assez rapidement qu'il s'agit d'une réunion de personnes appelées à se prononcer sur un conflit entre deux individus concernant un paiement pour services rendus. Je dis à notre observateur qu'il s'agit du fonctionnement d'un système juridique. En même temps, je lui explique que pour arriver à cette analyse, je suis passé à un niveau plus profond d'abstraction logique, c'est-à-dire au niveau (3), là où se situent les structures et les concepts échappant à la conscience des acteurs sociaux, mais culturellement spécifiques par rapport aux agencements psychiques universels. Lui, originaire d'une culture différente, peut faire -fera- une tout autre lecture, et quant au résultat, l'imagination n'a pas de bornes. Il peut y avoir pour lui solution de continuité en passant d'un niveau à un autre. Se mouvoir au sein de ce cadre exige donc des règles strictes de translation d'une case à une autre. La leçon à tirer de cet exemple simplifié à outrance est que les cases du cadre doivent être liées de façon insécable, mais que pour l'instant, répondre à cette exigence demande encore de la réflexion.

Il faut maintenant expliciter l'agencement tripartite en domaines de recherche. Soulignons en premier lieu que ces domaines sont aussi des domaines concrets. La distinction épistémologique entre nature, technique et culture est parfaitement fondée sur du réel. D'autres répartitions entre les domaines de recherche de phénomènes observés de la réalité vécue sont possibles, il est vrai, mais ceux-là ne peuvent correspondre qu'à des critères moins englobants que les nôtres. Tout d'abord, une distinction fondamentale partage les trois champs. Dans la nature, prise dans sa globalité et son universalité, l'entropie est négative. En revanche, les techniques sont 
caractérisées par une entropie positive (plus elle est positive plus nous dilapidons nos ressources en énergie) $)^{6}$. La notion même d'entropie est inopérante dans le domaine socioculturel. Dans la nature, les lois de l'évolution sont complexes, et linéaires -peu d'exemples de retour en arrière, bien que les convergences soient un phénomène courant. Dans le domaine du technique, l'évolution des processus et l'invention des outils obéissent plutôt à un régime cyclique, tantôt les techniques et le socioculturel évoluent pour ainsi dire séparément, tantôt les deux régimes sont plus intimement imbriqués et le social favorise l'innovation, ou au contraire bloque le changement. Dans les deux cas, les régularités observées relèvent d'une analyse faisant appel aux concepts de tendance et fait (Cresswell 1996 : chap. 9). Dans le champ du socioculturel il s'agit de système d'équilibres et de déséquilibres entre les modes d'adaptation des institutions sociales et du comportement individuel, d'abord entre eux, ensuite avec l'extérieur.

Venons-en au tableau des niveaux d'analyse. Soulignons que ce graphique n'est pas destiné à faire fonction d'outil de travail dans le sens strictement opérationnel du terme. En revanche, il s'agit bel et bien d'un cadre de recherche qui situe tant sur le plan épistémologique que sur un plan du réel concret (donc dans la logique et dans l'historie) les diverses études de technologie culturelle, les unes par rapport aux autres. Dans un premier rang, nous rangerons les éléments des études à faire, en deux mots les faits observables. Dans la colonne de la nature, ces éléments consistent essentiellement en matières premières. Quant aux techniques, nous placerons les acteurs et leurs outils -un artisan frappant avec son marteau sur une enclume, un technicien pressant un bouton qui fera démarrer les buses de peinture d'une carrosserie sur le pont. La troisième colonne contient des acteurs humains en interaction.

Dans un deuxième rang nous rangerons les assemblages des éléments observables : font partie du domaine de la nature, par exemple, les forces physiques, les formules chimiques; sont à ranger dans la colonne des techniques les types de produits, les réseaux de distribution; enfin les structures sociales, les entreprises, les institutions se retrouveront sous la rubrique « société ".

La différence entre ces deux rangs tient à ce que les éléments du premier n'ont pas entre eux de liens obligatoires. Un rabot, tant qu'il n'est pas utilisé, n'est une forme dans l'espace, et ni son nom ni sa place à côté d'un humain n'ont de réelle signification. En réalité, dans le signifiant "rabot» sont confondus deux niveaux d'étude: la description et l'analyse, la forme et la fonction. Il est vrai que cette façon de traiter les objets peut paraître inutilement formelle et statique, mais elle peut parfois servir à briser le carcan des idées reçues. Par exemple lorsqu'un objet en forme de hache est employé comme couteau de sculpteur (Leroi-Gourhan 1971). 


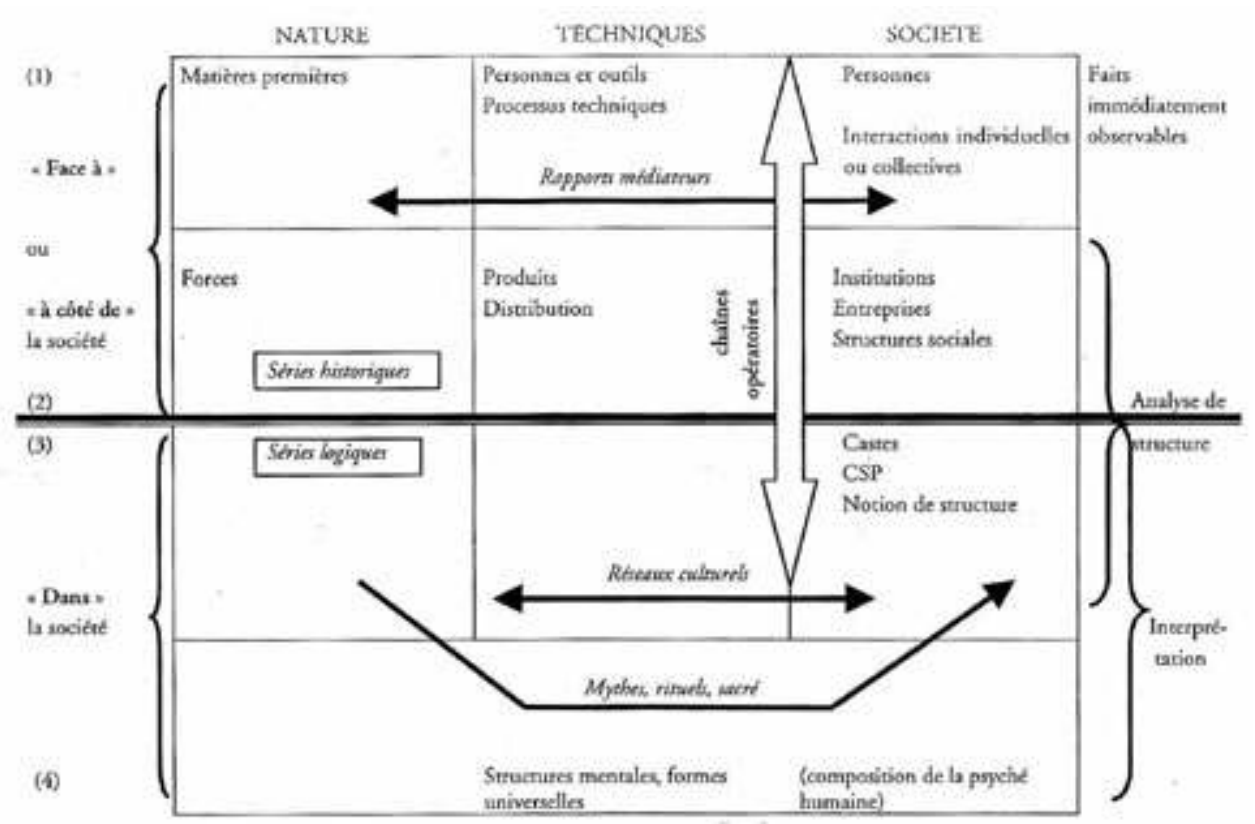

Figure 3. Les niveaux d'analyse

Le deuxième rang est celui de ce que l'on pourrait nommer l'analyse, car c'est le site où s'élaborent les relations et les liens, les processus et les structures visibles, voire les intentions et les usages invisibles -non visibles- au premier coup d'œil. Les phénomènes décrits et analysés dans ces deux premiers rangs constituent dans leur évolution et leur développement ce que j'appelle des séries historiques. C'est dire que leur existence est non seulement réelle, mais tangible. Elle est attestée dans les écrits ou dans l'iconographie, de même que leurs relations, leurs évolutions, en un mot leur histoire est consignée dans des restes physiques ou dans les mémoires. Sean, que j'observe en train de travailler la terre, est un être physique, tangible, qui occupe un espace inviolable. Je ne peux pas interposer les molécules de ma main dans l'espace occupé par les molécules de la sienne. Son corps est le siège de réactions biochimiques mesurables. Ses rapports avec ce qui l'entoure relèvent des mêmes réactions biochimiques. Lorsque je continue à l'observer, je le vois engagé dans un processus technique, ou interagir avec d'autres êtres humains dans, disons, un lieu qu'ils nomment «tribunal » où se dispense ce qu'ils appellent eux-mêmes la justice; je sais que je suis en présence de structures et de processus qui fonctionnent selon des règles connues et consultables, et dont l'histoire spécifique - peut-être fausse ou faussée, peu m'importe, la logique n'entre pas en ligne de compte, elle n'est en fait qu'accessoire à l'étude-.est attestée dans des écrits.

70 En revanche, en descendant au troisième rang, nous entrons dans ce que je nomme les séries logiques. D'un certain point de vue, elles correspondent aux taxa supraspécifiques des systématiciens. En effet, personne n'a jamais vu un Felis, mais tout le monde connaît l'une ou l'autre espèce classée dans ce genre, un ocelot, un chat de gouttière, un puma, par exemple. Je n'ai jamais vu une catégorie socio-professionnelle (CSP), à plus forte raison une classe sociale, mais j'ajuste mon comportement vis-à-vis des individus que je rencontre tous les jours aux règles des interactions entre ces catégories. 
71 La grande flèche indique que les chaînes opératoires servent de liens entre ces trois niveaux, du fait même que les techniques et les rapports sociaux sont "écrits " moyennant les mêmes termes.

72 Enfin, au niveau quatre où l'on risque peu de rencontrer des anthropologues - mais sûrement quelques psychanalystes égarés et, aujourd'hui une foule informe de cognitivistes et autres sbires de ce que l'on ne peut appeler qu'un mouvement postmoderne de "post-philosophie »-, se situent les structures mentales profondes de l'humanité, elles-mêmes probablement fondées sur l'organisation d'un psychisme commun à toute l'espèce humaine (dans le sens biologique du terme). On ne peut clore ces remarques sans évoquer les travaux de Bernard Juillerat (1991, 2001). Ce chercheur, dans une réflexion fondée sur des travaux de terrain exemplaires, définit les rapports entre anthropologie et psychanalyse. Cette discipline-ci va ranger dans notre quatrième niveau les quatre fantasmes originaires de Freud, le commerce sexuel des parents, la séduction, la castration, et le retour intra-utérin, ces fantasmes qui pour André Green jouent le rôle de matrice de l'inconscient (Green 1973, cité par Juillerat 2001: 56). Quant au cadre que je viens de proposer, l'hypothèse que cette matrice passant au troisième niveau active des fantasmes (dans les mythes entre autres) qui sont culturellement spécifiques, mais d'une certaine ressemblance, me paraît légitime. Comme le dit Juillerat, " ces diverses définitions sont susceptibles d'apporter des clés pour la compréhension des symboles culturels fournis par l'ethnographie » (2001). Naturellement, la réflexion sur cet aspect de l'étude des sociétés reste à approfondir.

Quant aux autres niveaux, le passage du premier au deuxième est œuvre d'observation et de classement d'objets qui apparaissent dans des séries historiques. Aller de celles-ci aux séries logiques est œuvre d'analyse informée. Ce qu'il faut trouver maintenant, ce sont les règles de passage entre rangs et colonnes. En ce qui concerne les techniques il faut parfaire l'élaboration de leur grammaire.

74 Dans la première partie de cet essai, les ramifications des problèmes soulevés par le titre et le sous-titre de cet ensemble d'articles furent examinées pour conclure qu la seule réponse aux questions posées était: «cela dépend!»Comme ont souvent répliqué mes informateurs à mes questions, sur mes trois terrains principaux, l'Irlande, le Liban, le Maroc. «C'est pour faire quoi ?» Ce type de réponse incertaine me paraît appeler impérativement l'emploi de l'outil de recherche qu'est la chaîne opératoire. Les manipulations et le traitement des matières premières sont des opérations où l'on est obligé de garder les pieds sur terre; et puisque les phénomènes sociaux et les gestes techniques sont inextricablement liés, l'espoir que la discussion que la discussion demeure dans le domaine de la réalité est fort.

75 J'ai ensuite esquissé un schéma qui laisse de la place au "selon les cas », car le cadre proposé assigne divers types d'approche aux différents aspects et niveaux où peut s'appréhender la réalité.

76 Pour terminer, je voudrais souligner que ces lignes relèvent bien plus d'un programme de travail en cours et à venir que d'un compte rendu de recherches accomplies. Je les adresse à l'ensemble des chercheurs en anthropologie. Quant à moi, c'est à son développement que j'ai l'intention de consacrer les années que me laisse l'usage sans doute imparfait que j'ai fait du temps écoulé. 


\section{BIBLIOGRAPHIE}

Amahan, Ali

1999. « Structures sociales et développement », pp 115-133, in Khrouz, Driss (ed.), Pour une approche alternative de l'analyse du développement. Casablanca : Fondation du Roi Abdul-Aziz Al Saoud pour les études islamiques et sciences humaines

Bunyan, John

1966. The Pilgrim's Progress. London : Oxford University Press (1678-1684).

Burke, James

1996. The Pinball Effect. New York : Little Brown.

Cresswell, Robert

1965. « Un pressoir à olives au Liban : essai de technologie comparée », L'Homme V (1) : 33-63. 1994. " La nature cyclique des relations entre le technique et le social. Approche technologique de la chaîne opératoire ", pp 275-289, in B. Latour et P. Lemonnier (eds), De la préhistoire aux missiles balistiques. L'intelligence sociale des techniques. Paris : La Découverte ("Recherches»). 1996. Prométhée ou Pandore, propos de technologie culturelle. Paris : Kimé.

2001. «La réalité et/de la nature. À propos de "l'affaire Sokal” ", L’Homme 157 : 175-196.

Juillerat, Bernard

1991. Edipe chasseur, une mythologie du sujet en Nouvelle Guinée. Paris : Presses Universitaires de France.

2001. Penser l'imaginaire. Essais d'anthropologie psychanalytique. Lausanne : Payot.

Korzybski, Alfred

2002. General Semantics. Brooklyn : Institute of General Semantics.

\section{NOTES}

1. Sur la notion de réalité dans les travaux d'anthropologie ou de sociologie $c f$. Cresswell 2001.

2. «Le bourbier de découragement».

3. Aujourd'hui dans les pays hautement industrialisés, près de trois-quarts de la population active travaille dans le secteur des services.

4. Des observations sur le jeu de go semblent indiquer que les joueurs humains appréhendent la stratégie à poursuivre par une visualisation globale des configurations possibles et souhaitables, ce qui les rend imbattables par les ordinateurs, lesquels travaillent en logique linéaire. L'intuition n'a manifestement pas encore dit son dernier mot. Il faudra peut-être réexaminer la notion d'intuition, à commencer par une relecture de Descartes.

5. Me permettra-t-on d'émettre une idée qui pourrait paraître à première vue de la plus haute fantaisie -j'en conviens-, mais qui à la longue -j'en suis convaincu- se révélera fructueuse ? Cette idée se résume en quelques phrases. Le propre de l'homme est de renverser l'entropie positive universelle, c'est donc, en un mot, d'introduire le l'ordre, de la hiérarchie, etc. Cela signifie structure, système, raisonnement et donc conflit, contradiction, paradoxe. L'analyse réflexive doit refléter cet état naturel des choses. Dans un raccourci que j'espère seulement saisissant, disons alors que le relativisme, le 
postmodernisme et tout déni d'une réalité en dehors de soi sont des efforts d'aplanissement antihumain.

6. Notons que cette entropie positive est un phénomène local, l'univers global affiche une entropie négative dans son parcours vers l'arrêt total.

\section{RÉSUMÉS}

Le titre de cet ensemble d'essais préconise trois thèmes. L'argument de cet article est que l'ordre dans lequel sont abordés ces thèmes est pertinent. De plus l'auteur énonce que la difficulté majeure découle de ce qu'à un certain niveau d'analyse, la technologie culturelle existe en tant que domaine séparé de la réalité sociale et ainsi appelle un champ autonome d'étude (ce qui implique une méthodologie spécifique), et qu'à un autre niveau d'analyse les techniques font structurellement partie intégrante de la société. Ainsi, les processus techniques et les structures sociales interagissent de deux manières. Pour prendre tout cela en compte, l'auteur propose non seulement d'agrandir la chaîne opératoire afin d'y inclure les canaux économiques de distribution et les interactions symboliques, mais aussi il suggère un cadre élaboré pour inclure les niveaux d'analyse et d'abstraction dans lequel pourraient prendre place les faits et les théories, l'action inconsciente et la signification inconsciente.

Technological act, a total social fact. Technics are in society, or confronting society?.

The title of this group of essays presupposes three themes. It is argued here that the order in which these are taken up is meaningful. In addition the author states that the essential difficulty is that on one level cultural technology sets out techniques as a separate domain of social reality and therefore calls for a autonomous field of study (which implies a distinctive methodology), and on another level technology is structurally an integral part of society. Thus technological processes and social structures interact in two ways. To take all this into account, the author proposes not only to enlarge the operative model of a work chain to include economic channels of distribution as well as symbolic interactions, but also suggests a framework consisting of levels of analysis and abstraction within which can be set fact and theory, conscious action and unconscious meaning.

INDEX

Mots-clés : chaîne opératoire, processus technique, structures sociales, méthodologie

Keywords : Methology, social structures, technical process, work chain

\section{AUTEUR}

\section{ROBERT CRESSWELL}

Directeur de recherche honoraire, Techniques et culture, UMR 8098, CNRS, 27 rue Paul Bert, 94204 Ivry-sur-Seine Cedex 